\title{
LOS VERBOS DE CAMBIO CON EL TIEMPO EN INGLÉS ANTIGUO ${ }^{1}$
}

ELBA VERA

Universidad de La Laguna

evera@ull.es

\section{RESUMEN}

Este artículo propone un sistema de descomposición léxica para los verbos de cambio con el tiempo en inglés antiguo que recoge el significado central de este subdominio y explica el comportamiento morfosintáctico de sus miembros de acuerdo con los principios del Modelo Léxico Construccional. De ahí que este trabajo suponga una descripción relativamente detallada de las últimas innovaciones dentro de este modelo y de cómo estas permiten una explicación satisfactoria de las peculiaridades de estos lexemas en inglés antiguo.

Palabras clave: Verbos de cambio con el tiempo, inglés antiguo, Modelo Léxico Construccional.

\section{ABSTRACT}

This paper presents a lexical analysis of the verbs of change in time in Old English that comprises information on the core meaning of this subdomain and the morphosyntactic behaviour of its members following the postulates of the Lexical Contructional Model. Consequently, it provides a detailed description of the last innovations wit-

${ }^{1}$ Esta investigación es parte del proyecto Arquitectura Semántica y Catálogo de Construcciones Sintáctico-Semánticas del Inglés Antiguo (HUM2005-07651-C02-01/FILO) financiado por el MEC. 
hin this frame and proofs of their proficiency in explaining the peculiarities of these historical lexemes.

KeY Words: Verbs of change in time, Old English, Lexical Constructional Model.

El Modelo Léxico Construccional (MLC; CORTES, 2007; MAIRAL y RUIZ DE MENDOZA, 2006; RUIZ DE MENDOZA y MAIRAL, 2007a,b...) surgió como una evolución natural del Modelo de Gramáticas Léxicas (MGL; MAIRAL, 2002 y 2003; MAIRAL y CORTÉS, 2002 y 2004; MAIRAL y FABER, 2002 y 2005...) tras un intento de establecer un medio de representación formado por elementos universales y solventar las incongruencias resultantes de usar una versión extendida del metalenguaje semántico de la Gramática del Papel y la Referencia (GPR; VAN VALIN y LAPOLLA, 1997; VAN VALIN, 2005).

De hecho, las propuestas en este sentido son una de las principales aportaciones de gran número de investigaciones en el marco de este modelo lexicológico (CORTÉS, 2007; MAIRAL y FABER, 2005; MAIRAL y RUIZ DE MENDOZA, 2006; RUIZ DE MENDOZA y MAIRAL, 2007a, b...2). En ellas se plantea una formalización abstracta diseñada mediante las estructuras lógicas de la GPR y una descripción semántica complementaria basada en las funciones léxicas de Mel'cuk (1996) y los primitivos semánticos del Metalenguaje Semántico Natural (MSN; GODDARD y WIERZBICKA, 2002).

Esta nueva herramienta descriptiva ha sido aplicada de forma parcial a numerosos dominios del inglés contemporáneo (verbos de contacto, existencia, cognición...); además de a los verbos de sonido (CORTÉS y GONZÁLEZ, 2006), la construcción impersonal en los verbos de felicidad (GARCÍA y VERA, 2006) y los verbos de disminución (VERA, 2007) en inglés antiguo. Sin embargo, el panorama en esta lengua histórica es un tanto exiguo cuando se toma en consideración el resto de innovaciones fundamentales del MLC.

Así, novedades teórico-descriptivas de máxima relevancia, como la introducción de un nuevo proceso restringido de subsunción en sustitución de las reglas léxicas y el proceso de modelaje, parecen no haber encontrado reflejo en los análisis de esta lengua llevados a cabo hasta el momento. De ahí la necesidad de llevar a cabo un estudio pormenorizado, no sólo del significado central del subdominio 'cambio con el tiempo' (To (cause) to change in time, FABER Y MAIRAL, 1999: 285) en inglés antiguo, sino también del comportamiento morfosintáctico de sus miembros.

Con este fin, la presente investigación parte de una breve introducción a los preceptos del MLC (primer apartado) para realizar un análisis pormenorizado de las propiedades semánticas y sintácticas de los verbos de cambio con el tiempo en inglés antiguo (apartados dos y tres, respectivamente) y explicitar los mecanismos de enlace que dan lugar a las configuraciones morfosintácticas reales de las oraciones en las que participan estos predicados verbales (apartado cuatro).

${ }^{2}$ Para un conocimiento más profundo de los trabajos y aplicaciones que se están realizando en el marco del Modelo Léxico Construccional, véase la página web del grupo LEXICOM (www.lexicom.es). 


\section{INTRODUCCIÓN AL MLC}

El primer paso para el estudio de cualquier dominio léxico según los preceptos del MLC es el establecimiento de una estructura lógica que sirva de soporte para la información sintácticamente relevante. Esta formalización es una representación de descomposición diseñada para describir la estructura semántica y argumental de un predicado en base a las distinciones de Aktionsart o ‘modo de acción'. Se trata de parámetros semántica y sintácticamente significativos para un gran número de lenguas, por lo que la clasificación de predicados verbales basada en ellos es evaluable mediante un conjunto de pruebas lingüísticas (VAN VALIN y LAPOLLA, 1997: 93-97).

El resultado de aplicar estos tests es una categorización cerrada de seis tipos verbales (VAN VALIN, 2005: 32-33) representados individualmente en forma de estructuras lógicas (VAN VALIN y LAPOLLA, 1997: 109; VAN VALIN, 2005: 45).

\begin{tabular}{|c|c|}
\hline Verb Class & Logical Structure \\
\hline State & predicate' $(\mathrm{x})$ or $(\mathrm{x}, \mathrm{y})$ \\
\hline Activity & do' $(\mathrm{x}$, [predicate' $(\mathrm{x})$ or $(\mathrm{x}, \mathrm{y})])$ \\
\hline Achievement & $\begin{array}{l}\text { INGR predicate' }(\mathrm{x}) \text { or }(\mathrm{x}, \mathrm{y}) \text {, or } \\
\text { INGR do' }(\mathrm{x},[\text { predicate' }(\mathrm{x}) \text { or }(\mathrm{x}, \mathrm{y})])\end{array}$ \\
\hline Semelfactive & $\begin{array}{l}\text { SEML predicate' }(x) \text { or }(x, y) \text {, or } \\
\text { SEML do' }(x \text { [predicate' }(x) \text { or }(x, y)])\end{array}$ \\
\hline Accomplishment & $\begin{array}{l}\text { BECOME predicate' }(\mathrm{x}) \text { or }(\mathrm{x}, \mathrm{y}) \text {, or } \\
\text { BECOME do' }(\mathrm{x},[\text { predicate' }(\mathrm{x}) \text { or }(\mathrm{x}, \mathrm{y})])\end{array}$ \\
\hline $\begin{array}{l}\text { ActiveAccomplishment } \\
\text { Causative }\end{array}$ & $\begin{array}{l}\text { do' }(\mathrm{x}, \text { [predicate1' }(\mathrm{x},(\mathrm{y}))]) \text { \& BECOME predicate2' }(\mathrm{z}, \mathrm{x}) \text { or }(\mathrm{y}) \\
\alpha \text { CAUSE } \beta \text {, where } \alpha, \beta \text { are representations of any type }\end{array}$ \\
\hline
\end{tabular}

Dado que estas pruebas lingüísticas han determinado que los predicados analizados son realizaciones o realizaciones causativas, se puede establecer las siguientes estructuras lógicas (VAN VALIN y LAPOLLA, 1997: 109) como los soportes fundamentales para describir pormenorizadamente este subdominio léxico:

BECOME predicate' $(\mathrm{x})$ or $(\mathrm{x}, \mathrm{y})$

[do' $(x, \varnothing)$ ] CAUSE [BECOME predicate' $(x)$ or $(x, y)]$

Sin embargo, las representaciones de los estados y las actividades, que sirven como base a toda estructura lógica, necesitan una mayor descomposición semántica para que éstas constituyan una forma de representación adecuada de las jerarquías léxicas tal y como las entiende el $\mathrm{MLC}^{3}$. De hecho, la naturaleza léxico-sintáctica de estos (sub)dominios hace necesaria la inclusión de un metalenguaje abstracto universal totalmente especificado y delimitado que permita la caracterización de todas y cada una de las peculiaridades de la clase léxica, independientemente de si estas tienen o no impacto sintáctico.

\footnotetext{
${ }^{3}$ Nótese que la primera descomposición semántica detallada es la que VAN VALIN Y WILKINS (1993) hacen del predicado remember y que VAN VALIN Y LAPOLLA (1997) llevan a cabo una labor similar con los verbos de actos de habla.
} 
Esta herramienta descriptiva incluye el conjunto de primitivos semánticos del MSN (GODDARD y WIERZBICKA, 2002) y una serie de operadores (las funciones léxicas de MEL'CUK, 19964) que permiten combinarlos para representar las peculiaridades específicas de sus respectivos hipónimos. De esta forma, a pesar de estar diseñadas para representar el conjunto de peculiaridades lingüísticas que caracterizan a un campo semántico, las plantillas léxicas también permiten describir a los distintos miembros de ese (sub)dominio (MAIRAL y FABER, 2002: 45, 51-54).

En ambos casos, la formalización generalizada de este sistema de representación mantiene la distinción entre su componente semántico y la estructura lógica a la que éste complementa (MAIRAL y RUIZ DE MENDOZA, 2006; RUIZ DE MENDOZA y MAIRAL, 2007a). Sin embargo, existe también una propuesta alternativa que favorece la incorporación de la información léxica y sintáctica en un único algoritmo (CORTÉS, 2007).

ANÁLISIS DE LOS VERBOS DE CAMBIO CON EL TIEMPO EN INGLÉS ANTIGUO

Así, según los preceptos del MLC, la plantilla léxica integrada del subdominio 'cambiar con el tiempo' en inglés antiguo es la siguiente:

[do' $(x, \varnothing)]$ CAUSE [BECOME other'(y) ProptContPlustime']

Esta descripción codifica cómo un realizador $x$ lleva a cabo una actividad indeterminada que hace que una entidad paciente $y$ sea distinta (other') con el tiempo. Este último matiz se manifiesta mediante el uso de una combinación de funciones léxicas, PROPTCONTPLus, susceptible de ser parafraseada como 'a causa de que continúe más', y el primitivo semántico time'.

Nótese que aunque la investigación llevada a cabo dentro de este modelo y sus predecesores ha permitido establecer los primitivos de todos y cada uno de los dominios verbales, Marial y Ruiz de Mendoza (2006b:4) reconocen explícitamente la singularidad de los dominios de EXISTENCIA y CAMBIO en lo tocante a la representación de los hipónimos mediante sus primitivos (be/happen y become, respectivamente). Esta dificultad se salva aquí de mediante el uso del primitivo other' del MSN, que en el contexto de las realizaciones parece ser la opción más adecuada para representar las propiedades de los verbos de CAMBIO.

En mi opinión, esto es así porque las realizaciones son un proceso no puntual con un final inherente que conlleva un estado resultante ${ }^{5}$ (VAN VALIN 2005: 42), lo que unido a un primitivo que expresa diferencia, hace que la representación BECOME other'(x) codifique un proceso que acaba necesariamente en un estado final distinto del inicial. In-

${ }^{4}$ Cabe destacar que en muchas de las últimas aportaciones en el marco del MLC (CORTÉS Y SOSA, 2008; MAIRAL Y RUIZ DE MENDOZA, 2008) se ha reemplazado estas funciones léxicas por las qualia de PUSTEJOVSKY (1995).

5 Da ahí que el operador característico de las realizaciones, BECOME, pueda descomponerse como PROC \& INGR, según VAN VALIN (2005: 44). 
dependientemente de si este último tiene o no representación léxica, la naturaleza específica de ese cambio (cfr. en apariencia, en cantidad, en polaridad, en temperatura, con el paso del tiempo...) se precisa mediante una combinación de funciones léxicas y/o primitivos semánticos, en el caso que nos ocupa ProptConTPlustime'.

La importancia de esta especificación viene dada, entre otros, por su trascendencia a la hora de describir los predicados que conforman el subdominio que describe la macrorrepresentación de la clase léxica cambio con el tiempo. No en vano, cada predicado individual que forma parte del (sub)dominio que aquí nos ocupa está descrito por expresiones derivadas de esta plantilla léxica canónica ${ }^{6}$.

CAMBIAR CON EL TIEMPO

Gomelian

Onealdian [BECOME other'(x) PROPTCONTPLustime']

Ripian [BECOME other' $\left(\mathrm{x}_{<\text {InvolvPredCulM }}\right)$ ProptContPlustime']

\section{HACER QUe Algo CAMbIE CON EL TIEMPO}

Ealdian [do' $(\mathrm{x}, \varnothing)]$ CAUSE [BECOME other'(y) ProptContPlustime']

[do' $(\mathrm{x}, \varnothing)$ ] CAUSE [BECOME other'( $\left.\mathrm{y}_{\text {<InvolvPredPeor }}\right)$ ProptContPlustime']

\section{Forealdian}

Geealdian [do' $(\mathrm{x}, \varnothing)]$ CAUSE [BECOME other'(y)ProptContPlustime']

Geripian $\quad[$ do' $(\mathrm{x}, \varnothing)]$ CAUSE [BECOME other' $\left(\mathrm{y}_{<I N V O L V P R D C U L M>}\right)$

ProptContPlustime']

Así, aparte de los previsibles predicados verbales inducidos y no inducidos con el significado genérico 'cambiar con el tiempo', este subdominio léxico contiene otros que se caracterizan por la especificación semántica de este valor. En concreto, los lexemas ripian y geripian presentan la peculiaridad de codificar como parte de su significado el hecho de que el argumento paciente alcance su culminación o apogeo (INVOLVPREDCULM).

(1) [BECOME other' (đīne æceras ${ }_{<\text {InvolvPRedCulm })}$ ) PROPTCONTPLustime']

$\begin{array}{lllllll}\text { Dō } & \text { đat } & \text { sunne } & \text { scine } & \text { đat } & \text { đìne aceras } & \text { rípion, }{ }_{\text {HoML TH. II. 104, 3. }} \\ \text { Hacer } & \text { que } & \text { sol } & \text { brillar } & \text { para que } & \text { tus campos } & \text { madurarSUBJ } \\ \text { IMPsg } & \text { CONJ } & \text { NOMsg } & \text { SUBJ } & \text { CONJ } & \text { NOM pl } & \text { PRES pl } \\ & & & & \text { PRES pl } & & \end{array}$

«Haz que el sol brille para que tus campos maduren»

${ }^{6}$ Este fenómeno se debe a la herencia de rasgos semánticos y sintácticos dentro de las clases léxicas. Sobre la justificación e importancia de esta transmisión de propiedades, véase Faber y Mairal (1999:107, 190, 199...), Mairal y Van Valin (2001), Mairal y Faber (2002) y Cortés y Mairal (2002). 
Frente a ellos, el archilexema inducido ealdian se caracteriza por poder especificar que al cambiar con el tiempo, la entidad aludida degenera (INVOLVPREDPEJOR) ${ }^{7}$.

(2) [BECOME other'(fultum heora ${ }_{<\text {InVolvPredPejor }>}$ ) PROPTCONTPLustime']

$\begin{aligned} & \text { Fultum heora } \quad \text { aldađ, } \\ & \text { Su S. SRт. 48, } 15 .\end{aligned}$
«Su ayda NOM sg decaer IND PRES sg
«Su decae con el paso del tiempo»

\section{CONSTRUCCIONES}

En cualquier caso e independientemente de su nivel de especificación, las descripciones particulares tienen un papel fundamental a la hora de explicar las diferentes realizaciones definitivas en las que aparecen los lexemas a los que representan. De hecho, en el marco del MLC, la interpretación semántica de las oraciones es el resultado de la subsunción restringida de las propiedades de las plantillas de los lexemas y de las construcciones en las que éstos participan.

En este sentido, cabe mencionar que los atributos de los patrones sintácticos se codifican mediante abstracciones de las partículas de significado sintácticamente relevantes extraídas de múltiples representaciones no genéricas (RUIZ DE MENDOZA y MAIRAL, 2007 : 4) y que éstas comparten el aparato formal de sus homólogas léxicas ${ }^{8}$. Es precisamente esta simetría la que permite la plena compatibilidad entre ambas descripciones, posibilitando su ensamblaje y, en consecuencia, la descripción de cualquier realización concreta de un predicado.

Esta compatibilidad formal no implica, sin embargo, una libertad absoluta de unión. De hecho, el mecanismo de subsunción propuesto por el MLC es de carácter explicativo e impone la exposición de las condiciones que fuerzan la creación de oraciones aceptables y el rechazo de aquellas consideradas inadmisibles.

En esta línea, los últimos avances de este modelo lexicológico (MAIRAL, 2003; MAIRAL y RUIZ DE MENDOZA, 2006; RUIZ DE MENDOZA y MAIRAL, 2007; CORTÉS, 2007) aportan un estudio preliminar de algunos ejemplos de los dos tipos posibles de limitaciones al ensamblaje. La primera categoría identificada, la de las restricciones internas, engloba a las disposiciones que hacen referencia a la dimensión semántica codificada en las plantillas léxicas y construccionales, a la naturaleza del primitivo o a las propiedades de las variables. De ahí que este tipo de restricciones se formulen en términos de las unidades metalingüísticas de la plantilla léxica (RUIZ DE MENDOZA y MAIRAL, 2007a: 4,15).

El otro orden, el de las restricciones externas, recoge, en cambio, todos aquellos mecanismos cognitivos que, aún siendo ajenos a las propiedades de las plantillas, condi-

\footnotetext{
${ }^{7}$ Nótese que esta propiedad semántica apunta a una posible relación semántica entre este predicado y los pertenecientes al subdominio 'Cambiar a peor', una dimensión de CAMBIO aún por analizar en esta lengua.

${ }^{8}$ Las variables internas, presentes en las plantillas léxicas e inexistentes en las representaciones de construcciones, suponen la única excepción a esta simetría (Ruiz de Mendoza y Mairal 2007b:11).
} 
cionan su subsunción (RUIZ DE MENDOZA y MAIRAL, 2007a: 4,15). Se trata, por tanto, de mecanismos bien delimitados como la metáfora y la metonímia (MAIRAL y RUIZ DE MENDOZA, 2006: 6-10; RUIZ DE MENDOZA y MAIRAL, 2007b: 5-8), supeditadas al Principio Extendido de Invariancia (RUIZ DE MENDOZA, 1998), el Principio de Correlación (RUIZ DE MENDOZA y SANTIBÁÑEZ, 2003) y el principio que rige la proyección entre dominios conceptuales (Mapping Enforcement Principle; RUIZ DE MENDOZA, 2005).

Independientemente de la acción y ámbito de estas restricciones, el proceso de subsunción consta, además, de dos variantes en función de la correspondencia o no entre las propiedades del predicado y de la construcción en la que éste participa (RUIZ DE MENDOZA y MAIRAL, 2007a: 13-15). Si esas cualidades no coinciden, se desarrolla un procedimiento de subsunción por conversión, que da lugar a patrones morfosintácticos que sólo pueden explicarse en función de la interacción entre las características de la construcción y las del predicado ${ }^{9}$ (RUIZ DE MENDOZA y MAIRAL, 2007a: 14-15).

Por el contrario, si existe una identificación perfecta entre las propiedades de la plantilla léxica y las de la construcción, se produce una subsunción por elaboración resultante en estructuras que provienen directamente de las cualidades del lexema. Es decir, nos encontramos ante situaciones en que los constituyentes de la realización textual son parte de la estructura argumental de la unidad: Bien porque la subsunción con la construcción establece la realización de elementos ya presentes en la plantilla léxica, o bien porque las propiedades de la construcción pueden derivarse de las del lexema (RUIZ DE MENDOZA y MAIRAL, 2007a: 13-14).

En el primer caso, la posibilidad elemental de elaboración es la identificación absoluta entre los componentes de la construcción y toda o parte de la plantilla léxica. Obviamente, este tipo de subsunción está regulado por la primera restricción interna reconocida por el MLC (MAIRAL y RUIZ DE MENDOZA, 2006: 10), aquella que establece la identidad plena de las variables, (sub)eventos y operadores de las plantillas.

Desafortunadamente, las peculiaridades propias del inglés antiguo, y de las lenguas históricas en general, hacen que los verbos de cambio con el tiempo sólo ofrezcan ejemplos de este fenómeno en dos verbos intransitivos, onealdian y ripian $^{10}$ :

(3) [BECOME other'(bān mine) PROPTCONTPLustime']

Onealdodon $\quad \begin{aligned} & \text { ān mine, } \text { Ps. SPL. 31,6. } \\ & \text { Envejecer IND } \\ & \text { mis huesos NOM pl pl }\end{aligned}$
PRET

«Mis huesos envejecen»

\footnotetext{
${ }^{9}$ Las muestras textuales existentes para los verbos de cambio con el tiempo en inglés antiguo no recogen ninguna evidencia de la acción de este proceso de subsunción, por lo que nos vemos obligados a posponer su tratamiento y ejemplificación para futuros estudios de esta lengua.

${ }^{10}$ El ejemplo transitivo que más se acerca a esta circunstancia es Mon sceal on eorpan geong ealdian. EXETER «Él/uno debe envejecer a los jóvenes de la tierra».
} 
(4) [BECOME other'(winberian $<$ InvolvPredCulm $)$ ProptCOnTPlustime']

$\begin{array}{llllll}\text { Hit } & \text { was } & \text { đa se tima } & \text { đe } & \text { winberian } & \text { ripodon. } \text {, }_{\text {соотеST }} \text {. } \\ \text { NOM sg } & \text { ser IND } & \text { el tiempo } & \text { cuando } & \text { uvas } & \text { madurar IND } \\ & \text { PRET pl } & \text { NOM sg } & \text { CONJ } & & \text { PRET pl }\end{array}$

«Era el tiempo en que las uvas maduraban»

Este panorama se invierte con la elaboración por identificación parcial que, en combinación con la condición que permite a las construcciones ser (sub)eventos de la plantilla léxicas de los predicados (MAIRAL y RUIZ DE MENDOZA, 2006: 11), caracteriza a la práctica totalidad de los lexemas inducidos de este subdominio. De hecho, como es habitual en el caso de las realizaciones causativas, la fusión de estos predicados ( $f o-$ realdian, geealdian y ealdian) con la construcción intransitiva supone su codificación como realizaciones no inducidas.

(5) [BECOME other'(bearn elelendisce) PROPTCONTPLustime']

$\begin{array}{ll}\text { Bearn elelendisce } & \text { forealdodon, }{ }_{\text {Ps. LAMB. } 17,46 .} \\ \text { Niños extranjeros } & \text { envejecer } \\ \text { NOM pl } & \text { IND PRET pl }\end{array}$

«Los niños extranjeros envejecieron»

(6) [BECOME other' (hiā) ProptContPlustime']

$\begin{array}{lllll}\text { Pte } & \text { hiā } & \text { lifia } & \text { and } & \text { gialdia, }{ }_{\text {RTL. 111, } 14 .} \\ \text { Que } & {\text { PRON } 3^{\text {a }}} & \text { vivir SUBJ } & \text { y } & \text { envejecer SUBJ } \\ \text { CONJ } & \text { sg }^{11} \text { NOM } & \text { PRES sg } & \text { CONJ } & \text { PRES sg }\end{array}$

«Que él vive y envejece»

(7) [BECOME other' (se faeder) ProptContPlustime']

\begin{tabular}{|c|c|c|c|c|c|}
\hline $\begin{array}{l}\text { Ponne } \\
\text { Cuando }\end{array}$ & $\begin{array}{l}\text { se sunu } \\
\text { el hijo }\end{array}$ & $\begin{array}{l}\text { wyxd, } \\
\text { crecer }\end{array}$ & entonces & $\begin{array}{l}\text { ealdad } \\
\text { envejecer }\end{array}$ & $\begin{array}{l}\text { se faeder, } \\
\text { el padre }\end{array}$ \\
\hline CONJ & NOM sg & IND PRES sg & ADV & AND PRES s & g NOM sg \\
\hline
\end{tabular}

Este fenómeno es, además, el ejemplo más evidente de la acción de las restricciones externas en el ámbito de este subdominio, ya que el proceso de elaboración por el que una realización causativa se reduce a realización no inducida está avalado por la metonímia PROCESS FOR ACTION (MAIRAL y RUIZ DE MENDOZA, 2006: 9-12; RUIZ DE MENDOZA y MAIRAL, 2007b: 15-17).

En esta misma línea, el proceso de elaboración por coincidencia de propiedades alternativo, aquel implica la determinación de las propiedades del lexema, tiene en este subdominio una aplicación especialmente significativa. Nos referimos a la intervención de gomelian en la variante intransitiva de la alternancia de sujeto poseedor (LEVIN, 1993: 77-8).

11 Variante del norte y Kéntica del pronombre hē, que puede ser tanto nominativo singular como plural (BOSWORTH y TOLLER 1898: 511). 
No en vano, la fusión con esta construcción impone una especificación del significado de este lexema focalizando el efecto de lo descrito en una parte o cualidad de la entidad paciente:

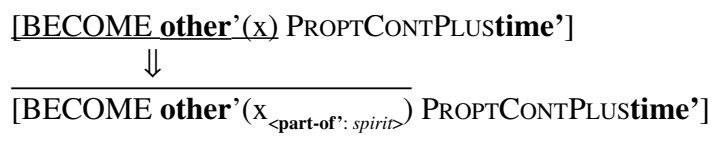

A pesar de la escasez de evidencias y lo reducido del ámbito de este análisis, la realización de este argumento adjunto parece ser un punto de encuentro entre el inglés contemporáneo (LEVIN, 1993: 77-8) y su antecesor. Ambos presentan sintagmas introducidos por la preposición in como único medio de codificar el atributo o fracción de la entidad paciente en la que se evidencia la realización descrita por gomelian.

Sin embargo, a pesar de esta semejanza, no debe obviarse que, a diferencia del inglés contemporáneo (LEVIN, 1993: 78), la lengua anglosajona presenta pacientes animados ${ }^{12}$.

(8) [BECOME other' $\left(\right.$ God $\left._{<\text {part-of': spirit }>}\right)$ PROPTCONTPLUStime']

$\begin{array}{llll}\text { Ne } & \text { gomelad } & \text { God } & \text { in gäeste, }{ }_{\text {GN. Ex. II. }} \\ \text { No ADV } & \text { envejecer IND } & \text { Dios NOM sg } & \text { en espíritu } \\ & \text { PRES sg } & & \text { PREP + DAT sg }\end{array}$

«Dios no envejece en espíritu»

(9) [BECOME other' $\left(h e_{<\text {part-of': spirit }}\right)$ PROPTCONTPLUStime']

$\begin{array}{llll}\text { Ne } & \text { gomelad } & \text { he } & \text { in gaste, }{ }_{\text {COEXETER }} \\ \text { No ADV } & \text { envejecer IND } & \text { PRON } 3^{\text {a }} \mathrm{sg} & \text { en espíritu } \\ & \text { PRE sg } & \text { NOM } & \text { PREP + DAT sg }\end{array}$

«Él no envejece en espíritu»

\section{ENLACE}

Una vez que se han obtenido y explicado las estructuras resultantes del proceso de subsunción, en su afán explicativo, el MLC recurre el algoritmo de enlace de la GPR (VAN VALIN y LAPOLLA, 1997: capítulo 7 y 9) para justificar la configuración morfosintáctica real de las oraciones.

El primer elemento de este mecanismo es la identificación de las generalizaciones gramaticalmente relevantes de los tipos de argumentos presentes en los predicados (VAN VALIN, y LAPOLLA, 1997: 139). Es decir, la vinculación de las funciones de macrorrol activo (Actor) y/o pasivo (Undergoer) con los argumentos en una plantilla según la jerarquía universal que gobierna tanto dicha asignación como el grado de prototipicidad de la misma (VAN VALIN y LAPOLLA, 1997: 146; VAN VALIN, 2005: 51).

\footnotetext{
${ }^{12}$ Nótese que estas oraciones presentan, además, signos evidentes de una identificación entre ciertos subeventos de la plantilla léxica y la construcción, por lo que ejemplifican la acción de las restricciones internas ya expuestas con anterioridad.
} 


\begin{tabular}{lllll} 
& & \multicolumn{3}{c}{} \\
\cline { 3 - 5 } Arg. of & $1^{\text {st }}$ arg. of & $1^{\text {st }}$ arg. of & $2^{\text {nd }}$ arg. of & Arg. of state \\
DO & doo $^{\prime}(x, \ldots$ & pred $^{\prime}(x, y)$ & pred $^{\prime}(x, y)$ & pred $^{\prime}(x)$
\end{tabular}

[ ' $\longrightarrow$ ' = increasing markedness of realization of argument as macrorole]

De este modo, la asignación de macrorroles canónica para los verbos de cambio con el tiempo en inglés antiguo se explica en función de la localización de los argumentos con respecto al predicado al que complementan y del tipo de éste (Default Macrorole Assignment Principles; VAN VALIN y LAPOLLA, 1997: 152-153, VAN VALIN, 2005: $52-53)$.

a. Number: the number of macroroles a verb takes is less thau or equal to the number of arguments in its logical structure,

1. If a verb has two o more arguments in its LS, it will take two macroroles.

2. If a verb has one argument in its LS, it will take one macrorole.

b. Nature: for the verbs which take one macrorole,

1. If the verb has an activity predicate in its LS, the macrorole is Actor.

2. If the verb has no activity predicate in its LS, the macrorole is undegoer.

Así, en las oraciones en que el predicado verbal es complementado por un solo argumento, éste, dado el carácter no dinámico del evento, funciona siempre como macropapel pasivo:

(10) [BECOME other' (bān mīne $\left.e_{\text {UNDERGOER }}\right)$ PROPTCONTPLUstime']

Onealdodon

bān mīne, Ps. SpL. 31, 6 .

Envejecer IND

mis huesos

PRET pl

NOM pl

«Mis huesos envejecen»

(11) [BECOME other'(bearn elelendisce UNDERGOER ) PROPTCONTPLUStime']

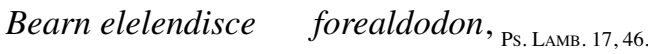

Niños extranjeros envejecer IND

NOM pl PRET pl

«Los niños extranjeros envejecieron»

(12) [BECOME other' $\left(\right.$ hi $\left.\bar{a}_{\text {UNDERGOER }}\right)$ PROPTCONTPlustime']

$\begin{array}{lllll}\text { Pte } & \text { hiā } & \text { lifia } & \text { and } & \text { gialdia, }{ }_{\text {RtL. 111, 14. }} \\ \text { Que } & \text { PRON 3 }^{\text {a }} & \text { vivir } & \text { y CONJ } & \begin{array}{l}\text { envejecer } \\ \text { CONJ }\end{array} \\ \text { sg NOM } & \text { SUBJ } & & \text { SUBJ PRES sg } \\ & & \text { PRES sg } & & \end{array}$

«Que él vive y envejece» 
(13) [BECOME other' (se faeder ${ }_{\text {UNDERGOER }}$ ) PROPTCONTPLustime']

$\begin{array}{llllll}\text { Ponne se sunu } & \text { wyxd, } & \text { ponne } & \text { ealdad se faeder, } \\ \text { Cuando el hijo } & \text { crecer } & \text { entonces } & \text { envejecer el padre } \\ \text { CONJ } & \text { NOM } & \text { IND PRES } & \text { sg } & \text { IND PRES NOM sg } \\ & \text { sg } & \text { sg } & \text { ADV } & \text { sg }\end{array}$

«Cuando el hijo crece, el padre envejece»

(14) [BECOME other'(winberian $<$ INvolvPredCulm>Undergoer $)$ ProptCONTPlustime']

Hit was đase tima đe winberian ripodon. COотеST$^{\text {. }}$

PRON $3^{\text {a }}$ ser IND el tiempo cuando uvas NOM pl madurar IND

sg NOM PRETsg NOMsg CONJ PRET pl

«Era el tiempo en que las uvas maduraban»

(15) [BECOME other'(fultum heora ${ }_{<\text {InvolvPRedPejor>UNDERgoer }}$ ) PROPTCONTPlustime']

Fultum heora aldađ, ${ }_{\text {Ps. SRT. } 48,15 .}$

Su ayuda NOM sg decaer IND PRES sg

«Su ayuda decae con el paso del tiempo»

Esta asignación se repite incluso cuando una construcción impone la especificación de la parte de la entidad paciente en la que se focaliza el cambio:

(16) [BECOME other' $\left(\right.$ God $\left._{<\text {part-of': spirit>UNDERGOER }}\right)$ PROPTCONTPLUStime']

$\begin{array}{llll}\text { Ne } & \begin{array}{l}\text { gomelad } \\ \text { envejecer IND } \\ \text { No ADV }\end{array} & \text { God } & \text { Dios } \\ & \text { PRES sg } & \text { NOM sg } & \text { in ente, } \text { Gn. Ex. II. }^{\text {espitu }} \\ & \text { PREP + DAT sg }\end{array}$

«Dios no envejece en espíritu»

(17) [BECOME other' $\left(h e_{<\text {part-of': spirit> UNDERGOER }}\right)$ PROPTCONTPLUStime']

$\begin{array}{llll}\text { Ne } & \begin{array}{l}\text { gomelad } \\ \text { No ADV ejecer IND }\end{array} & \text { he } & \text { in gaste, } \\ & \text { PRES sg } & \text { PRON } 3^{a} \text { sg NOM. II. } & \text { en espíritu } \\ & \text { PREP + DATsg }\end{array}$

«Él no envejece en espíritu»

De hecho, esa falta de dinamicidad se hace aún más evidente cuando el pronombre sylf funciona como mero enfatizador del sintagma nominal de la oración principal ${ }^{13}$ :

(18) [BECOME other' $\left(s y l f_{<\text {InvolvPredPejor>Undergoer }}\right)$ ProptCONTPLustime']

$\begin{array}{lllll}\text { Sele } & \text { sceal } & \text { stondan, } & \text { sylf } & \text { ealdian, } \\ \text { Bueno } & \text { tener que IND } & \text { permanecer } & \text { él mismo } & \text { decaer } \\ \text { NOM sg } & \text { PRES sg } & \text { INF } & \text { PRON } & \text { INF }\end{array}$

«El bueno ha de permanecer, de decaer con el tiempo»

${ }^{13}$ Sobre este uso del pronombre sylf, véase Mitchell (1985: §495-§496). 
Este carácter no dinámico y la consecuente asignación de macropapeles tiene, además, consecuencias inmediatas en la asignación de la función de Argumento Sintáctico Privilegiado mediante la jerarquía (VAN VALIN, y LAPOLLA, 1997:282) dispuesta a tal efecto:

Arg.of DO $>1^{\text {st }} \arg$. of do' $>1^{\text {st }} \arg$. of pred' $(x, y)>2^{\text {nd }} \arg$. of pred' $(x, y)>\arg$. of pred' $(x)$

$\mathrm{Al}$ ser una lengua acusativa, el inglés antiguo asigna por regla general este estatus al elemento con función semántica o macropapel más alto en la escala (VAN VALIN, y LAPOLLA, 1997:282; VAN VALIN, 2005:95). Así, la ausencia de realizadores que funcionen como macrorroles activos supone que la entidad paciente que desempeña el macropapel secundario sirve también como controlador de las oraciones intransitivas de nuestro corpus.

(19) [BECOME other' (bān mīne $e_{\text {UNDERGoER:PSA }}$ ) ProptCONTPlustime']

Onealdodon $\quad$ bān mīne, ${ }_{\text {Ps. SPL. 31, } 6 .}$
Envejecer IND $\quad$ mis huesos
PRET pl NOM pl
«Mis huesos envejecen»

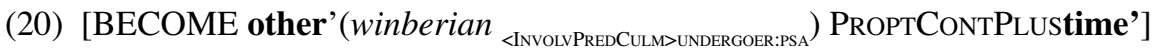
Hit was đase tima đe winberian ripodon. PRON $3^{\text {a }}$ ser IND el tiempo cuando uvas NOM pl madurar IND sg NOM PRETsg NOMsg CONJ PRET pl «Era el tiempo en que las uvas maduraban»

(21) [BECOME other' (fultum heora $a_{\text {<INVOLYPRDPEIOR>INDERGoER:PSA }}$ ) ProptContPlustime']

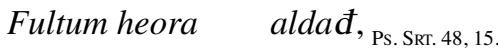
Su ayuda NOM sg decaer IND PRES sg «Su ayuda decae con el paso del tiempo»

(22) [BECOME other' $\left(\operatorname{God}_{\langle\text {part-of': spiriti UNDERGOER:PSA }}\right)$ PROPTCONTPLUStime']

$\begin{array}{llll}\text { Ne } & \begin{array}{l}\text { gomelađ } \\ \text { No ADV }\end{array} & \text { God } & \text { in gäejecer IND } \\ & \text { enve. Ex. II. } & \text { Dios } & \text { en espíritu } \\ & \text { PRES sg } & \text { NOM sg } & \text { PREP + DAT sg }\end{array}$

«Dios no envejece en espíritu»

Esta asignación se mantiene incluso en el caso de las oraciones pasivas, cuyos realizadores o agentes, en el caso de existir, tendrían un rango inferior a la entidad que realiza las funciones de controlador.

(23) [BECOME other' $\left(I c_{\text {UNDERgoer: PSA }}\right)$ PrOPTCONTPlustime']

$\begin{array}{llll}\text { Ic } & \text { eom } & \text { forealdod } & \text { betweoh eallum minum feondum. copRrPs } \\ \text { PRON } 1^{\text {a }} & \text { ser IND } & \text { envejecer } & \text { entre todos mis enemigos } \\ \text { NOM sg } & \text { PRES sg } & \text { PART } & \text { PREP + DAT pl }\end{array}$

«Me envejezco entre mis enemigos» 
(24) [BECOME other' $\left(\mathrm{x}_{\text {UNDERGOER: PSA }}\right)$ PROPTCONTPLustime']

Geealdad

Envejecer PART

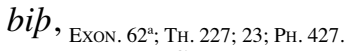

«Se envejece»

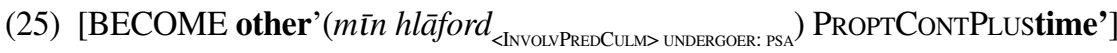

$\begin{array}{lll}\text { Mīn hläford } & \begin{array}{l}\text { geripod } \\ \text { envejecer PART }\end{array} & \begin{array}{l}y s, \text { GEN. 18, 12 } \\ \text { ser IND PRES sg }\end{array} \\ \begin{array}{ll}\text { Mi señor NOM sg } & \end{array} & \end{array}$

Sea cual fuere, la asignación de macropapeles proporciona la identificación de las categorías morfosintácticas características de los constituyentes centrales no oblicuos. Para ello, ha de seguirse el siguiente conjunto de principios (VAN VALIN, y LAPOLLA, 1997:359; 368; VAN VALIN, 2005: 95):

Case assignment rules for accusative constructions:

a. Assign nominative case to the highest-ranking macrorole argument (in terms of the privileged syntactic argument selection hierarchy).

b. Assign accusative case to the other macrorole argument.

c. Assign dative case to non-macrorole arguments (default).

Así, el algoritmo de enlace y las reglas de asignación de caso permiten hallar las representaciones semánticas totalmente especificadas de las estructuras morfosintácticas finales de las oraciones en las que participan los verbos de cambio con el tiempo:

(26) [BECOME other'( $b a \bar{n}$ minne $\left.e_{\text {UNDERGOER:PSA } \rightarrow \text { NOM }}\right)$ PROPTCONTPLUStime']

Onealdodon bān mīne, ${ }_{\text {Ps. SpL. 31, } 6 .}$

Envejecer IND mis huesos

PRET pl NOM pl

«Mis huesos envejecen»

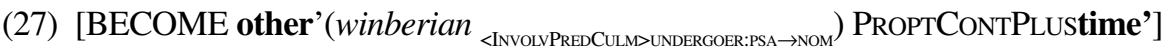
Hit was đase tima đe winberian ripodon. соотеsт $^{\text {. }}$ PRON $3^{\text {a }}$ ser IND el tiempo cuando uvas NOM pl madurar IND sg NOM PRETsg NOMsg CONJ PRET pl

«Era el tiempo en que las uvas maduraban»

(28) [BECOME other'(fultum heora ${ }_{<\text {INvolvPredPejor>UnDergoer:PSADNom }}$ ) ProptContPlustime']

Fultum heora aldad, ${ }_{\text {Ps. SRT. } 48,15 .}$

Su ayuda NOM sg decaer IND PRES sg

«Su ayuda decae con el paso del tiempo» 


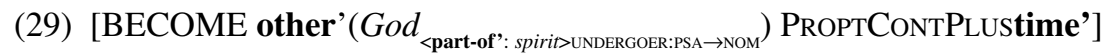

$\begin{array}{llll}\text { Ne } & \text { gomelad } & \text { God } & \text { in gāeste, }{ }_{\text {GN. Ex. II. }} \\ \text { No ADV } & \text { envejecer IND } & \text { Dios } & \text { en espiritu } \\ & \text { PRES sg } & \text { NOM sg } & \text { PREP + DAT sg }\end{array}$

«Dios no envejece en espíritu»

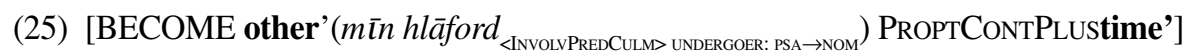
Mìn hläford $\begin{array}{ll}\text { geripod } & y s,{ }_{\text {Gen. } 18,12} \\ \text { envejecer PART } & \text { ser IND PRES sg }\end{array}$ Mi señor NOM sg envejecer PART ser IND PRES sg «Mi señor se envejecía»

Además de su acción descriptiva, estas representaciones tienen el valor añadido de ser el primer requisito para establecer la interficie semántico-sintáctica de los verbos de cambio con el tiempo ${ }^{14}$. No en vano, basta con satisfacer el Principio de Selección de Plantillas Sintácticas (VAN VALIN, y LAPOLLA, 1997: 173) y preservar las relaciones de prominencia (Argument Prominence Constraint y Structural Prominence Constraint; MAIRAL, 2002: 78) para proceder a la explicación pormenorizada de las conexiones entre las distintas representaciones semánticas y sintácticas de las realizaciones textuales de los verbos de cambio con el tiempo.

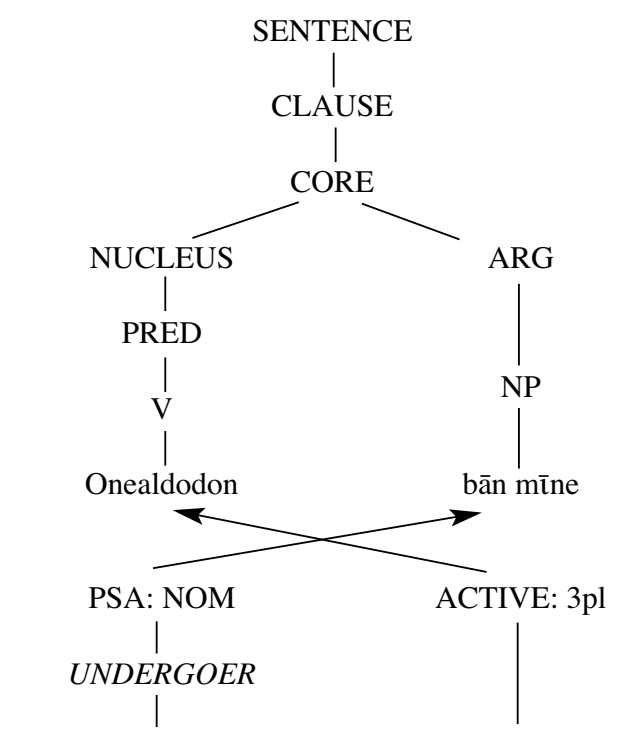

[BECOME other' (bān mīne) PRoPTConTPLustime']

Onealdodon bān mīne, PS. SpL. 31,6 .

«Mis huesos envejecen»

${ }^{14}$ Con este fin, el antecesor inmediato del MLC, el MGL, postulaba un algoritmo de enlace (MAIRAL, 2002: 74). Sin embargo, este mecanismo carece de correlato alguno en el modelo actual, en el que el proceso de subsunción restringida determina toda interacción entre las plantillas léxicas y las construcciones. 


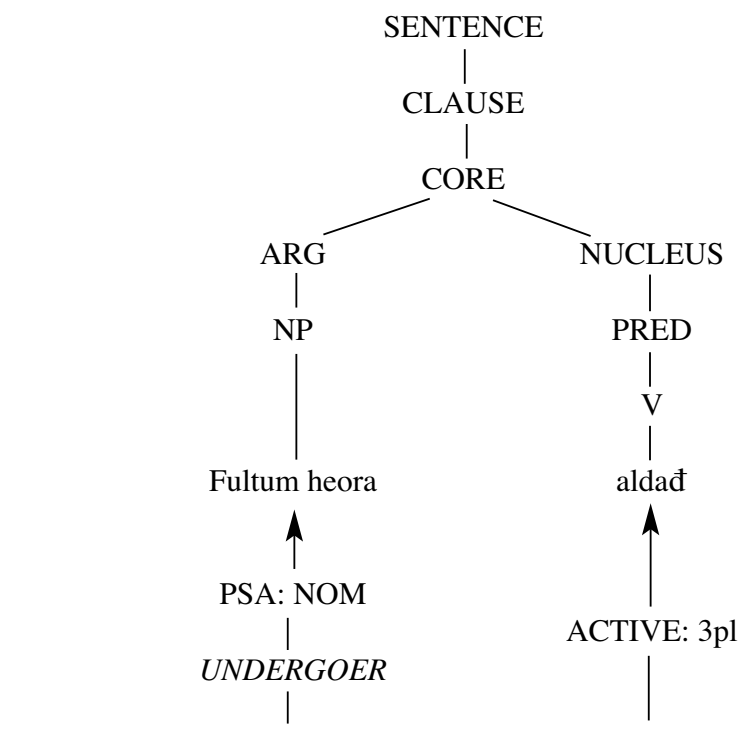

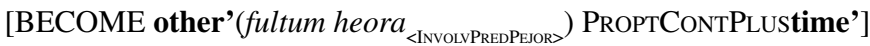

Fultum heora aldađ, ${ }_{\text {Ps. SRr. } 48,15 .}$

«Su ayuda decae con el tiempo»

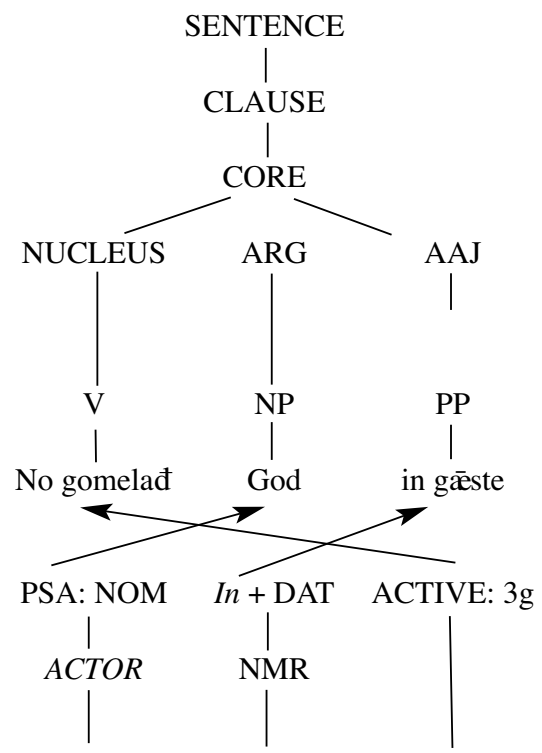

[BECOME other' $\left(\right.$ God $\left._{<\text {nart-of' } \text { spirit }}\right)$ PROPTCONTPLustime']

No gomelađ God in gaéste, GN. Ex.II.

«Dios no envejece en espíritu» 


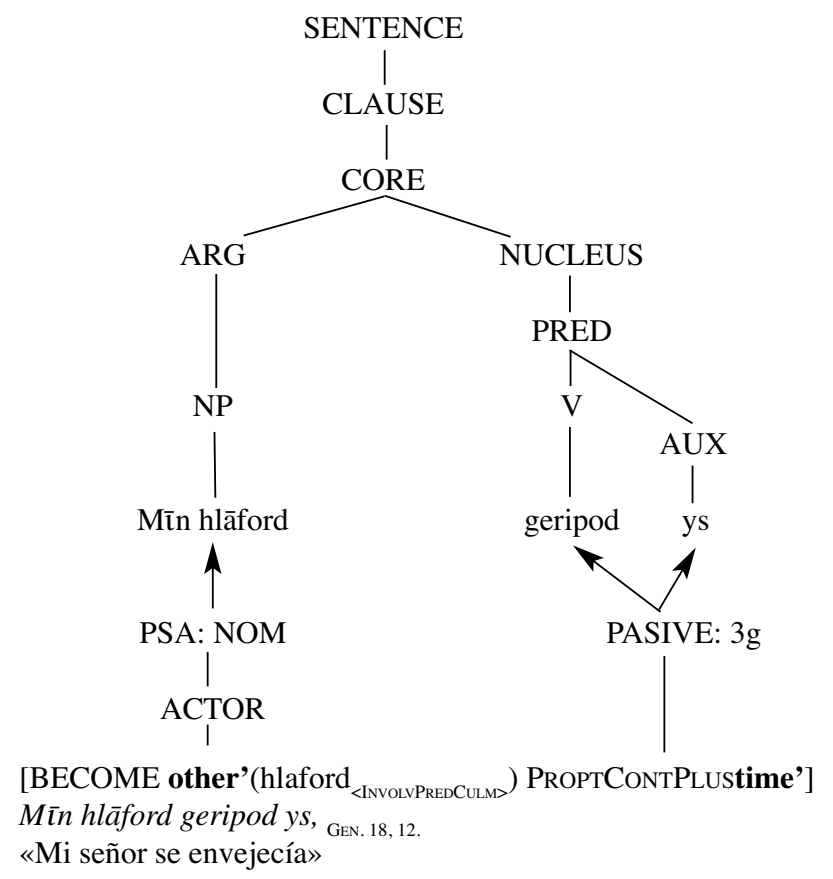

\section{CONCLUSIONES}

En su conjunto, estos enlaces suponen una explicación de las propiedades semánticas y sintácticas de las oraciones en las que participan los predicados pertenecientes al subdominio de cambio con el tiempo. Una exposición que unida a las descripciones de las propiedades semánticas y sintácticas de este campo léxico y las peculiaridades de sus constituyentes que la preceden supone un estudio muy pormenorizado de esta clase léxica.

Tanto es así, que la presente investigación podría considerarse incluso una evidencia del acierto del MLC al combinar ciertos elementos de sus predecesores con nuevos mecanismos aún más explicativos para lograr un entendimiento profundo del léxico verbal anglosajón.

\section{REFERENCIAS BIBLIOGRÁFICAS}

Bosworth, Joseph y Toller, T. Northcote (1898): An Anglo-Saxon Dictionary. Reimpresión 1972. Londres: Oxford University Press.

CORTÉs, Francisco José (2007): Building a Constructicon: Templates, Constraints and Unification. Trabajo inédito de investigación.

CoRTÉs, Francisco José y GonZÁlez, Marta María (2006): «Anglo-Saxon Verbs of Sound: Semantic Architecture, Lexical Representation and Constructions». Studia Anglica Poznaniensia 42: 249-284. 
CoRTÉs, Francisco José y MaIRAL, Ricardo (2002): «A Preliminary Design for a Syntactic Dictionary of Old English on Semantic Principles» en Díaz Vera, J. ed. A Changing World of Words. Ámsterdam y Nueva York: Rodopi, 3-46.

CORTÉs, Francisco José y SosA, Eulalia (2008): «The Morphology-semantics Interface in Word Formation» Revista Canaria de Estudios Ingleses 57, noviembre 2008:91-108.

FABER, Pamela. B. y MAIRAL, Ricardo (1999): Constructing a Lexicon of English Words. Berlín y Nueva York: Mouton de Gruyter.

GARCíA, Carmen Dolores y VerA, Elba (2006): «The Lexical Representation of the Impersonal Construction in Old English Verbs of Happening» 2006 Role and Reference Grammar International Conference. Leipzig.

Goddard, Ciff y WiERZBicka, Anna (2002): Meaning and Universal Grammar: Theory and Empirical Findings. Ámsterdam y Filadelfia: John Benjamins.

Levin, Beth (1993): English Verb Classes and Alternations: A Preliminary Investigation. Chicago: Chicago University Press.

MAIRAL, Ricardo (2002): Lexical Templates and Syntactic Structures: The Design of a Lexical Grammatical Model. Trabajo inédito de investigación. Madrid: UNED.

- (2003): «Reconsidering the Syntax-Semantics Interface in Role and Reference Grammar» en Celada, A. R.; D. Pastor García y P.J. Pardo García, eds. 2003. Actas del XXVII Congreso Internacional de AEDEAN. Salamanca: Ambos Mundos.

MAIRAL, Ricardo y CORTÉs, Francisco José (2002): «Representaciones Semánticas y Estructuras Sintácticas: Propuestas de Interrelación entre el Léxico y la Gramática» en Mairal, R. y Pérez, $\mathrm{M}^{\mathrm{a}} \mathrm{J}$. eds. New Perspectives on argument structure in Functional Grammar. Berlín y Nueva York: Mouton de Gruyter.

- (2004): «Rethinking Lexical Representation in Role and Reference Grammar» en Kastousky, D. ed. The English Lexicon: Structure and Genesis. Berlín y Nueva York: Mouton de Gruyter.

MAIRAL, Ricardo y FABER, Pamela B. (2002): «Functional Grammar and lexical templates» en Mairal, R. y M $\mathrm{M}^{\mathrm{a}} \mathrm{J}$. Pérez eds. New Perspectives on argument structure in Functional Grammar. Berlín y Nueva York: Mouton de Gruyter.

- (2005): «Decomposing Semantic Decomposition. Towards a Semanti Metalanguage in RRG». 2005 Role and Reference Grammar International Conference. Taiwan.

MAIRAL, Ricardo y RUIZ DE MENDOZA, Francisco José (2006): «Internal and external constraints in meaning construction: the lexicon-grammar continuum» en Estudios de Filología Inglesa: Homenaje a la Dra. Asunción Alba Pelayo. Madrid: UNED.

- (2008): «New Challenges for Lexical Representation within the Lexical-constructional Model (LCM)» Revista Canaria de Estudios Ingleses 57, noviembre 2008:137-158.

MAIRAL, Ricardo y VAN VALIN, Robert D. Jr. (2001): «What Role and Reference Grammar can do for Functional Grammar» en Pérez Quintero, $\mathrm{M}^{\mathrm{a}}$. J. ed. Challenges and Developments in Functional Grammar. Revista de Estudios Ingleses 42, abril 2001: 137-166.

MeL'CuK, Igor A. (1996): «Lexical Functions: A Tool for the Description of Lexical Relations in a Lexicon» en Wanner, L. Lexical Functions in Lexicography and Natural Language Processing. Ámsterdam y Filadelfia: John Benjamins. 37-102.

Mitchell, Bruce (1985): Old English Syntax, volume I: concord, the parts of speech and the sentence. Oxford: The Clarendon Press.

Pustejovsky, James (1995): The Generative Lexicon. Cambridge, Mass.: MIT Press.

RuIZ DE MENDOZA, Francisco José (1998): «On the nature of blending as a cognitive phenomenon». Journal of Pragmatics 30(3): 259-274.

- (2005): «High level cognitive models: in search of a unified framework for inferential and grammatical behavior» en Kosecki, K. ed. Perspectives on Metonimy. Frankfurt y Main: Peter Lang. 
Ruiz De Mendoza, Francisco José y SantibáÑEz, Francisco (2003): «Content and formal cognitive operations in construing meaning». Italian Journal of Linguistics 15(2): 293-320.

RuIZ de Mendoza, Francisco José y MaIRAL, Ricardo (2007b): «Levels of semantic representation: where lexicon and grammar meet». Interlingüística 17: 26-47.

- (2007a): «High level metaphor and metonymy in meaning construction» en Radden, G.; K-M. Köpcke; T. Berg y P. Siemund eds. Aspects of Meaning Construction in Lexicon and Grammar. Ámsterdam y Filadelfia: John Benjamins. 33-49.

VAN VALIN, Robert D. Jr. (2005): Exploring the Syntax-Semantics Interface. Cambridge: CUP.

VAN VAlin, Robert D. Jr. y LAPOLla, Randy (1997): Syntax: Structure, Meaning and Function. Cambridge: CUP.

- (1997): Syntax: Structure, Meaning and Function. Cambridge: CUP.

VerA, Elba (2007): «La Representación Léxica de Disminuir en Inglés Antiguo». XXXI Congreso Internacional AEDEAN. A Coruña. 